\title{
Characterization of semiconductor nanowires using optical tweezers.
}

\begin{abstract}
We report on the optical trapping characteristics of InP nanowires with dimensions of $30( \pm 6)$ $\mathrm{nm}$ in diameter and $2-15 \mu \mathrm{m}$ in length. We describe a method for calibrating the absolute position of individual nanowires relative to the trapping center using synchronous high-speed position sensing and acousto-optic beam switching. Through Brownian dynamics we investigate effects of the laser power and polarization on trap stability, as well as length dependence and the effect of simultaneous trapping multiple nanowires.
\end{abstract}

Keyword: Semiconductor nanowires; Optical tweezers; Brownian motion; Nanoparticle metrology. 